\title{
Production of Metal-Free Diamond Nanoparticles
}

\author{
Laia Ginés, ${ }^{*} \dagger \odot$ Soumen Mandal, ${ }^{\dagger}$ David John Morgan, ${ }^{\ddagger \odot}$ Ryan Lewis, ${ }^{\S}$ Philip R. Davies, ${ }^{\ddagger}$ Paola Borri, ${ }^{\S}$ \\ Gavin W. Morley," and Oliver A. Williams* ${ }^{\dagger}$ \\ ${ }^{\dagger}$ School of Physics and Astronomy, Cardiff University, The Parade, Cardiff CF24 3AA, U.K. \\ ${ }^{\ddagger}$ Cardiff Catalysis Institute, School of Chemistry, Cardiff University, Main Building, Park Pl, Cardiff CF10 3AT, U.K. \\ ${ }^{\S}$ School of Biosciences, Cardiff University, The Sir Martin Evans Building, Museum Avenue, Cardiff CF10 3AX, U.K. \\ "Department of Physics, University of Warwick, Coventry CV4 7AL, U.K.
}

Supporting Information

ABSTRACT: In this paper, the controlled production of high-quality metal-free diamond nanoparticles is demonstrated. Milling with tempered steel is shown to leave behind iron oxide contamination which is difficult to remove. Milling with $\mathrm{SiN}$ alleviates this issue but generates more nondiamond carbon. Thus, the choice of milling materials is critically determined by the acceptable contaminants in the ultimate application. The removal of metal impurities, present in all commercially available nanoparticles, will open new possibilities toward the production of customized diamond nanoparticles, covering the most demanding quantum applications.

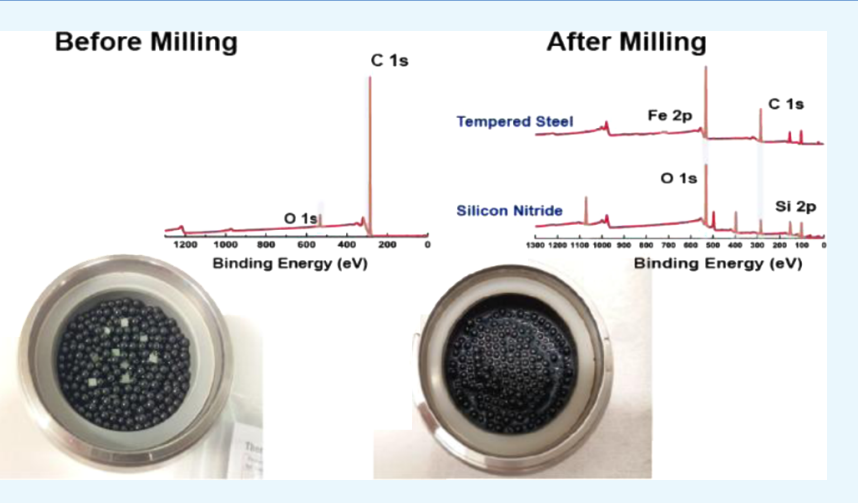

are detrimental for biological applications. Additionally, commercial nanodiamonds typically have a high concentration of nitrogen defects $[\mathrm{N}]>100 \mathrm{ppm}$, which reduces $\mathrm{NV}^{-}$spin coherence times and hence magnetic field sensitivity. ${ }^{26}$ This high nitrogen content is also a problem for the levitation of diamond nanoparticles in high vacuum as the nitrogen absorbs the trapping laser light, heating and burning the diamond nanoparticles. $^{27,28}$ Furthermore, a non-negligible amount of metal contaminants ${ }^{29,30}$ is also present in commercially available nanoparticles, reducing the possibilities of the magnetic-related applications. Inductively coupled plasma mass spectrometry studies performed by Volkov et al. ${ }^{29}$ over 20 different commercial detonation diamond particles detected high amounts of metal impurities in all of them.

For these reasons, it is highly important to be able to create customized diamond particles. Several methods for particles' production are known up to date. The most versatile one is the production of diamond nanoparticles by crushing chemical vapor deposition (CVD) diamond or bulk diamond using milling techniques. ${ }^{31,32}$ This approach enables particle size distribution control and offers the possibility of creating custom color centers as well as particles from bulk diamond with low nitrogen concentration. Nevertheless, deep cleaning methods are required after the milling process.

Received: August 16, 2018

Accepted: November 8, 2018

Published: November 28, 2018 
In this paper, the production of metal-free particles from commercial single-crystal (SC) bulk diamond is shown. Different milling materials are compared.

\section{EXPERIMENTAL METHODS}

Commercial CVD grown SC diamond samples, $0.3 \mathrm{~mm}$ thick $(2.6 \mathrm{~mm} \times 2.6 \mathrm{~mm}$ sized) sourced from Element Six, were used in this study. Two different grinding bowls, one made from tempered steel and one made from silicon nitride, were used for crushing the SC plates. Twelve SC plates (95 mg approximately) were introduced in each grinding bowl with 5 $\mathrm{mL}$ of deionized (DI) water and 40 and $30 \mathrm{~g}$ of tempered steel and silicon nitride grinding balls $(d=3 \mathrm{~mm})$, respectively. Samples were milled in the Planetary Micro Mill PULVERISETTE 7, following 6 cycles of $5 \mathrm{~min}$ on/15 min off at 1100 rpm ( $95 \mathrm{~g})$. After the milling process, the samples were cooled down and taken out of the grinding bowls and several acid cleaning processes were performed. For iron removal, the cleaning was performed as described by Heyer et al. ${ }^{32}$ For the silicon nitride cleaning, $20 \mathrm{~mL}$ of sample was mixed with 30 $\mathrm{mL}$ of orthophosphoric acid $\left(\mathrm{H}_{3} \mathrm{PO}_{4}\right)$ and the mixture was stirred continuously in a condenser during $24 \mathrm{~h}$ at $180{ }^{\circ} \mathrm{C}$ bath temperature. To remove the acids, both solutions underwent repeated washing and centrifugation cycles at $30000 \mathrm{~g}$, removing the supernatant after each centrifugation process and adding DI water to the pellet until the $\mathrm{pH}$ reached a value between 5.8 and 6.0. The slurries were dried in a hot plate to obtain the powders. For the silicon nitride grinding process, the obtained powder was introduced again in the condenser after being dissolved in $20 \mathrm{~mL}$ of water, and $30 \mathrm{~mL}$ of concentrated sodium hydroxide $(\mathrm{NaOH})$ solution was added. The mixture was stirred in the condenser during $24 \mathrm{~h}$ at 150 ${ }^{\circ} \mathrm{C}$. The washing and centrifugation cycles were repeated as previously described until $\mathrm{pH} 5.8-6$ was reached. After the cleaning and centrifugation cycles, the tempered steel-milled and the silicon nitride-milled powders were treated in a furnace under air atmosphere at $600{ }^{\circ} \mathrm{C}$ for $5 \mathrm{~h}$. Different aqueous colloids were prepared from the treated powders by dispersing $0.01 \mathrm{~g}$ of powder in $20 \mathrm{~mL}$ of DI water. The colloids were dispersed via ultrasound, and the solutions were centrifuged at different accelerative forces $(5000 \mathrm{~g}, 10000 \mathrm{~g}, 20000 \mathrm{~g}$, and 30 $000 \mathrm{~g})$ at $10{ }^{\circ} \mathrm{C}$ in a Sigma 3-30 KS centrifuge. Dynamic light scattering (DLS) and nanoparticle tracking analysis (NTA) measurements were performed to measure the particles' size distribution. The Malvern Zetasizer Nano ZS used in our experiments was equipped with a $633 \mathrm{~nm}$ laser in backscattering configuration $\left(173^{\circ}\right)$ and the Malvern NanoSight LM10 was equipped with a $635 \mathrm{~nm}$ laser.

Raman measurements were recorded in an inVia Renishaw confocal Raman microscope equipped with a $532 \mathrm{~nm}$ laser. All the measurements were acquired using the same parameters: $10 \mathrm{~s}$ acquisition time and 50 accumulations.

For comparison of the surface chemistry of each powder, Xray photoelectron spectroscopy (XPS) measurements were performed in a Thermo Scientific K-Alpha ${ }^{+}$spectrometer. Spectra were acquired using a monochromatic $\mathrm{Al}$ source operating at $72 \mathrm{~W}(6 \mathrm{~mA}$ emission current $\times 12 \mathrm{kV}$ anode potential). A survey and a high-resolution spectrum were acquired at pass energies of 150 and $40 \mathrm{eV}$, respectively. Charge neutralization was achieved using the K-Alpha charge neutralization system, employing a combination of both electrons and low energy argon ions. All XPS spectra were calibrated with the carbon $\mathrm{C} 1 \mathrm{~s}$ peak at $285 \mathrm{eV}$.

\section{RESULTS AND DISCUSSION}

XPS measurements were taken in various steps. (1) On pristine SC substrate, (2) after tempered steel milling, (3) after

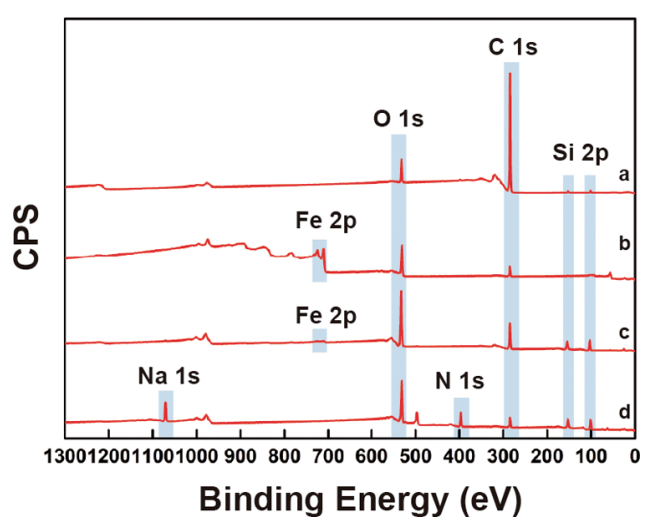

Figure 1. Survey XPS spectrum of the different samples. (a) SC raw material just before the milling, (b) powder after milling using tempered steel grinding bowl, (c) powder after the tempered steel milling and the acid cleaning to remove the metal contaminants, and (d) powder after the silicon nitride milling.

tempered steel milling followed by acid cleaning, and (4) after the silicon nitride milling followed by its corresponding acid cleaning treatment (see Experimental Methods). Figure 1 shows the XPS survey for the mentioned processes with all the detectable elements present in the samples.

Figure 1a shows the XPS spectrum of the as-received SC substrate. The XPS spectrum shows two clear peaks corresponding to carbon ( $\mathrm{C} 1 \mathrm{~s}$ peak) and oxygen ( $\mathrm{O} 1 \mathrm{~s}$ peak) elements at 285 and $531.8 \mathrm{eV}$, respectively. A small third peak ( $\mathrm{Si} 2 \mathrm{p}$ ) also appears at lower binding energies. No silicon should be present in the sample, and so the sample was subjected to argon cluster cleaning. The complete removal of the silicon after the cluster cleaning indicates some kind of surface contamination. (see Figure S1). The survey XPS spectrum in Figure $1 \mathrm{~b}$ corresponds to the sample milled with the tempered steel milling bowl and balls. The spectrum shows four elements: carbon (C 1s), oxygen ( $\mathrm{O} 1 \mathrm{~s})$, iron ( $\mathrm{Fe} \mathrm{2p}$ ), and silicon $(\mathrm{Si} 2 \mathrm{p})$. The Fe $2 \mathrm{p}$ peak confirms the presence of iron in the sample produced in the milling process. Although acid cleaning was performed in order to remove all the metallic components, non-negligible amounts of iron were detected (XPS detection limit is $10 \mathrm{ppm}$ ) after the acid cleaning, as shown in Figure 1c. The Si $2 \mathrm{p}$ peak presence can be neglected as this silicon is due to contamination present previous to the milling process and can be removed with argon cluster etching inside the XPS chamber.

$\mathrm{C} 1 \mathrm{~s}, \mathrm{O} 1 \mathrm{~s}, \mathrm{Si} 2 \mathrm{p}, \mathrm{N}$ 1s, and $\mathrm{Na} 1 \mathrm{~s}$ peaks are present in the SC sample milled with silicon nitride (shown in Figure 1d).

In this case, the Si $2 \mathrm{p}$ and $\mathrm{N}$ 1s peaks are related to silicon and nitrogen contamination because of insufficient cleaning of the silicon nitride produced in the milling. The $\mathrm{Na} 1 \mathrm{~s}$ peak is due to sodium contamination in the cleaning process, as $\mathrm{NaOH}$ was used in the cleaning. Different cleaning methods for $\mathrm{Si}_{3} \mathrm{~N}_{4}$ removal can be found in the literature. Kim et al. ${ }^{33}$ proposed $\mathrm{Si}_{3} \mathrm{~N}_{4}$ dissolution utilizing an $\mathrm{HF}, \mathrm{H}_{2} \mathrm{SO}_{4}$, and $\mathrm{HNO}_{3}$ mixture. Nevertheless, although $\mathrm{Si}_{3} \mathrm{~N}_{4}$ was not completely removed, iron ( $\mathrm{Fe} 2 \mathrm{p}$ peak) was not detected in the sample milling with silicon nitride, making the use of a silicon nitride 


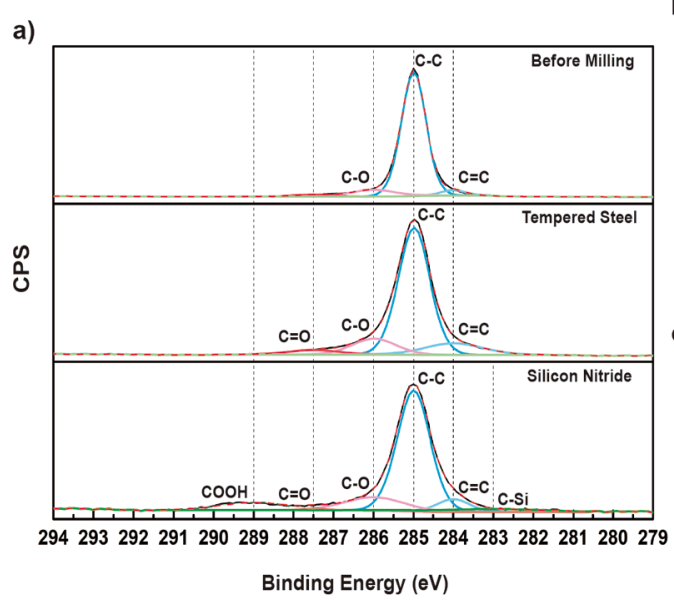

b)

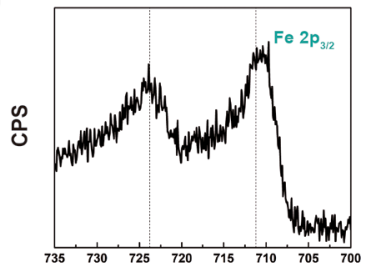

Binding Energy (eV)

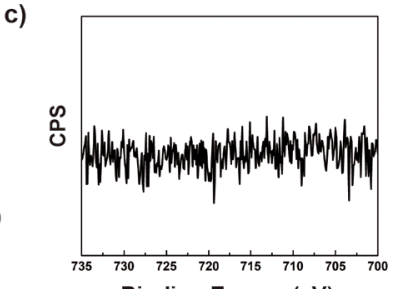

Binding Energy (eV)

Figure 2. High-resolution XPS scans for the carbon and iron elements. (a) Carbon (C 1s) deconvoluted peak for the as-received SC, and the powders after been milled with the tempered steel and the silicon nitride grinding bowls, respectively. (b) Iron (Fe 2p) peak for the SC samples milled in a tempered steel grinding bowl and (c) Fe $2 p$ peak of the SC sample milled in the silicon nitride grinding bowl.

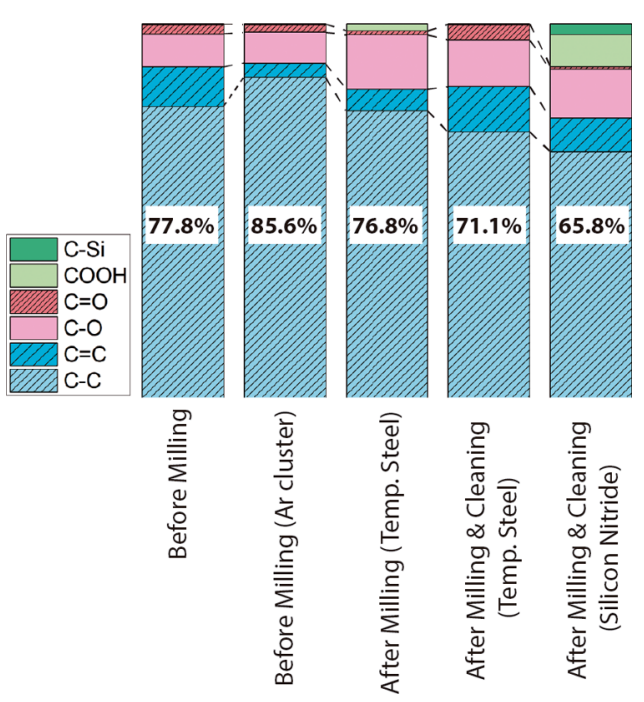

Figure 3. Bar plot with the percentages of the relative contents of the $\mathrm{C} 1 \mathrm{~s}$ peak for the different samples.
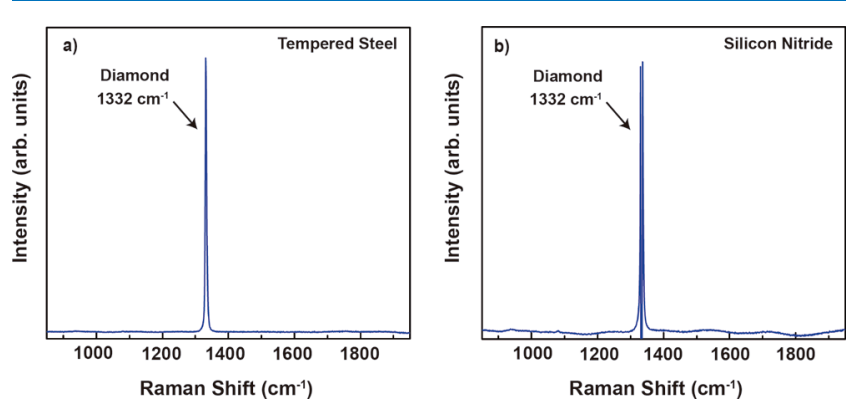

Figure 4. Raman measurements of the powders after the milling, acid cleaning, and air annealing processes. (a) SC powder after tempered steel milling process and (b) SC powder after the silicon nitride milling process.

grinding bowl extremely important in magnetometry applications.

The atomic percentages of the different elements present in the samples can be calculated from the survey spectrum, after the normalization of the peak areas considering the appropriate sensitivity factors (see Table S1). ${ }^{34}$ A drastic increase in the $\mathrm{O}$ 1s/C 1s ratio is observed after the milling processes because the samples are subjected to an air annealing (oxidation) treatment after the respective acids cleaning treatments. Further increase in the $\mathrm{O} 1 \mathrm{~s} / \mathrm{C} 1 \mathrm{~s}$ ratio was obtained for the SC sample milled with the silicon nitride.

To obtain further information about the elements present in the samples, high-resolution scans were performed in the region of interest. The data were fitted using Gaussian fits in CasaXPS, after subtraction of a Shirley type background.

All the peaks present in the XPS survey were analyzed in detail (Figures S2-S4), but only the carbon and the iron spectra are discussed in this paper. In Figure 2, high-resolution scans for the $\mathrm{C} 1 \mathrm{~s}$ and the iron $\mathrm{Fe} 2 \mathrm{p}$ peaks of the different samples are shown. The fitting of the $C 1$ s peak shows different components with small binding energy (BE) shifts between them. However, there is no consensus in the literature to assign each $\mathrm{BE}$ to a component, as different $\mathrm{BE}$ values have been reported for the same chemical species. ${ }^{35-38} \mathrm{BE}$ shifts of -1 , $+1,+2.5$, and $+4 \mathrm{eV}$ were taken from the literature ${ }^{39}$ and assigned to graphitic carbon, hydroxyl $(\mathrm{C}-\mathrm{OH}) /$ ether $(\mathrm{C}-$ $\mathrm{O}-\mathrm{C})$, carbonyl $(\mathrm{C}=\mathrm{O})$, and carboxyl groups $(\mathrm{COOH})$. An additional shift of $-3 \mathrm{eV}$ was considered for the silicon nitridemilled sample, corresponding to $\mathrm{Si}-\mathrm{C}$ bonds. ${ }^{40,41}$

The $\mathrm{C} 1 \mathrm{~s}$ peak for the SC sample before any milling process shows a symmetric spectrum with a dominant peak at $285 \mathrm{eV}$, attributed to the $\mathrm{C}-\mathrm{C}$ bond ( $\mathrm{sp}^{3}$ bonded carbon). In the same graph, small contributions attributed to both $\mathrm{C}=\mathrm{C}$ bonds and $\mathrm{C}=\mathrm{O}$ bonds were also observed at lower and higher binding energies, respectively. The SC diamond sample milled with tempered steel presents, however, a slightly asymmetric peak consisting of four Gaussian peaks, centered at 284, 285, 286, and $287.5 \mathrm{eV}$. The first peak can be assigned to $\mathrm{sp}^{2}$ carbon $(\mathrm{C}=\mathrm{C})$. The peak at $285 \mathrm{eV}$ corresponds to $\mathrm{sp}^{3}$ bonded carbon, the peak at $286 \mathrm{eV}$ is attributed to $-\mathrm{C}-\mathrm{H} / \mathrm{C}-\mathrm{O}$ bonds, and the peak at $287.5 \mathrm{eV}$ is assigned to the $\mathrm{C}=\mathrm{O}$ bonds. $^{42}$

The SC sample after the silicon nitride milling presents, in contrast, an asymmetric peak with a tail toward higher binding energies, which indicates a higher $\mathrm{sp}^{2}$ carbon concentration. Apart from the peaks described for the tempered steel sample, two more peaks are clearly observed. 

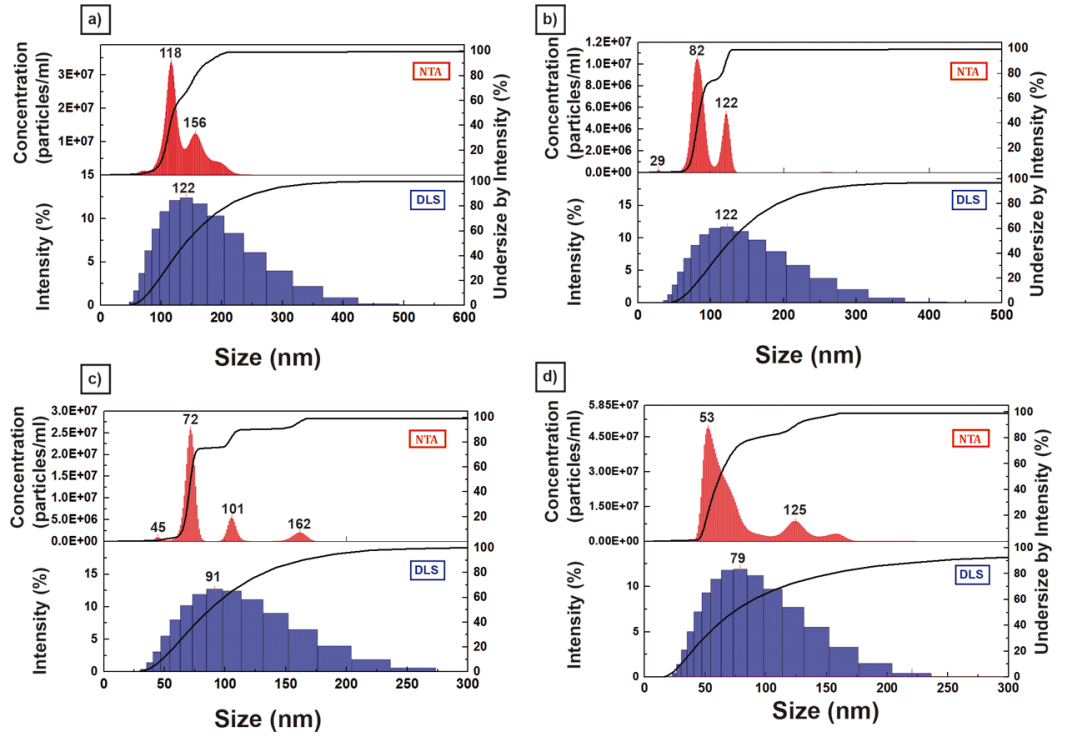

Figure 5. Particles' size distribution of the tempered steel-milled powders' solution after centrifugation at different accelerative forces: (a) 5000g; (b) $10000 g$; (c) $20000 g$; and (d) $30000 g$.

The peak at the lowest energy, $283 \mathrm{eV}$, confirms the presence of carbon bonded to silicon, and the peak at $289 \mathrm{eV}$ can be assigned to carboxyl groups $(\mathrm{COOH})$ or to the $\pi-\pi^{*}$ transition. ${ }^{43,44}$

The differences between the three samples are more pronounced representing the percentage of the relative contents as shown in Figure 3. The sample before the milling process subjected to the cluster argon ion cleaning has also been included for comparison.

A reduction in the $\mathrm{sp}^{3}(\mathrm{C}-\mathrm{C})$ content is clearly observed between the as-received sample, the as-received sample subjected to an argon cluster cleaning (85.6\%), and the sample milled with the silicon nitride material (65.8\%). In the latter sample, the appearance of the $\mathrm{COOH}$ and $\mathrm{C}-\mathrm{Si}$ components is evident.

Clear differences in the fitting of the $\mathrm{C} 1$ s peak are observed, showing further graphitization produced with the silicon nitride milling.

Whereas the study of the $\mathrm{C} 1 \mathrm{~s}$ peak can provide information about the surface graphitization ( $\mathrm{sp}^{2}$ content), the analysis of the Fe $2 p$ peak will confirm the presence of undesired metal impurities. Iron was detected in the survey spectra of the SC diamond sample milled with the tempered steel. A highresolution scan for the Fe $2 p$ peak is shown in Figure $2 \mathrm{~b}$. The $\mathrm{BE}$ of the $\mathrm{Fe} 2 \mathrm{p}_{3 / 2}$ peak was observed at $711.2 \mathrm{eV}$, which corresponds to the core level spectra of $\mathrm{Fe}^{3+}$ ions. ${ }^{45}$ Although iron was not detected in the SC sample milled with the silicon nitride, a more detailed scan for the Fe $2 p$ peak was also performed for this sample for comparison. Figure $2 c$ shows the XPS measurement of this peak in which iron was not detected.

Even though silicon nitride milling is the best method to avoid any metal content in the diamond particles produced, it also has some drawbacks. The silicon nitride material generated in the milling process is difficult to remove. Also, the asymmetry in the $C$ is peak confirms higher surface graphitization of the particles when compared to the tempered steel-milled sample. The $\mathrm{sp}^{2}$ carbon, either in the form of graphitic-like carbon or amorphous carbon, is detrimental for many applications. Attempts to remove the $\mathrm{sp}^{2}$ carbon by conducting an air annealing treatment ${ }^{46}$ at $600{ }^{\circ} \mathrm{C}$ did not result in the complete removal of $\mathrm{sp}^{2}$ carbon.

To confirm the quality of the diamond powders obtained after crushing the SC plates, Raman measurements were performed on the samples.

Figure 4 shows the Raman measurements for the different SC powders. A sharp and clear diamond peak centered at 1332 $\mathrm{cm}^{-1}$ can be observed in the samples milled with both tempered steel (Figure 4a) and silicon nitride (Figure $4 b$ ). Furthermore, a small band between 1500 and $1600 \mathrm{~cm}^{-1}$, known as the G-band, can be distinguished in the sample milled with the silicon nitride (Figure 4b), confirming the presence of $\mathrm{sp}^{2}$ sites. $^{47}$

The possibility of selecting and controlling the particles' size, highly important for different applications, represents an enormous advantage over commercial nanoparticles. Particles with sizes below $70 \mathrm{~nm}$ are desired for quantum applications, with particles as small as $10 \mathrm{~nm}$ containing active NV centers. ${ }^{48}$ Furthermore, particles with sizes between 50 and $100 \mathrm{~nm}$ are also suitable for drug delivery bio-applications. ${ }^{49}$ Particle size distributions can be controlled by combining longer milling times and colloids centrifugation at higher accelerative forces. Figure 5 shows the particle size distributions in the colloids made from tempered steel-milled particles as described in the experimental section. Two different characterization methods, DLS and NTA, as well as different accelerative forces to select particle size distributions were used.

In polydispersed colloids, it is important to use various characterization methods. For instance, in DLS, the presence of particles with various sizes can lead to imprecise particle size distributions. This is due to the fact that in DLS, the particle size is determined from intensity fluctuations in the Rayleigh scattering off a volume of the particles. As the intensity of Rayleigh scattering is proportional to $d^{6}$, where $d$ is the particle diameter, large particles or aggregates can mask the measurement of smaller particles. On the other hand, NTA gives a more precise measure as individual particles can be tracked. Figure 5a shows the distribution of particles after centrifugation at $5000 \mathrm{~g}$. In the NTA analysis graph, two different particle 
size distribution peaks can be differentiated, 118 and $156 \mathrm{~nm}$, whereas the particle size distribution is broader for the DLS measurement. This difference increases with increasing centrifuge accelerative force as seen in Figure $5 b-d$. Three particle size distributions were distinguished at $10000 \mathrm{~g}$ (Figure $5 b), 29,82$, and $122 \mathrm{~nm}$, but in the DLS measurement a mean value of $122 \mathrm{~nm}$ was obtained. Centrifugation at higher revolutions per minute results in smaller fractions of particles with large diameters, and particle size distribution down to 53 $\mathrm{nm}$ was recorded after centrifugation at $30000 \mathrm{~g}$.

\section{CONCLUSIONS}

In summary, diamond nanoparticles with controlled sizes have been produced following two distinct milling strategies. Highquality starting material and the choice of the grinding bowl material will ultimately determine the subsequent potential applications.

Milling with the tempered steel material results in $\mathrm{Fe}_{2} \mathrm{O}_{3}$ presence in the diamond nanoparticles even after the acid cleaning process, which excludes their use in magnetic/spinrelated applications. Silicon nitride milling is a good choice to ensure metal-free diamond nanoparticles, but results in larger nondiamond contamination, difficult to remove. Although the silicon nitride milled process showed the presence of sodium, the hydroxide cleaning process can be discarded in favor of producing metal-free nanoparticles.

\section{ASSOCIATED CONTENT}

\section{S Supporting Information}

The Supporting Information is available free of charge on the ACS Publications website at DOI: 10.1021/acsomega.8b02067.

XPS extended data and sample compositions in atomic percentages (PDF)

\section{AUTHOR INFORMATION}

\section{Corresponding Authors}

*E-mail: GinesL@cardiff.ac.uk (L.G.).

*E-mail: WilliamsO@cardiff.ac.uk (O.A.W.).

\section{ORCID $\odot$}

Laia Ginés: 0000-0001-9980-054X

David John Morgan: 0000-0002-6571-5731

\section{Author Contributions}

The manuscript was written through contributions of all authors. All authors have given approval to the final version of the manuscript.

\section{Notes}

The authors declare no competing financial interest.

\section{ACKNOWLEDGMENTS}

L.G., S.M., and O.A.W. would like to thank the Royal Society International Exchanges Scheme (IE131713) and EU FP7 FET Open "Wavelength tunable Advanced Single Photon Sources". XPS data collection was performed at the EPSRC National Facility for XPS ("HarwellXPS"), operated by Cardiff University and UCL, under contract no. PR16195. GWM is supported by the Royal Society and the EPSRC (EP/J014664/ 1).

\section{REFERENCES}

(1) Tang, L.; Tsai, C.; Gerberich, W. W.; Kruckeberg, L.; Kania, D. R. Biocompatibility of Chemical-Vapour-Deposited Diamond. Biomaterials 1995, 16, 483-488.

(2) Thomson, L. A.; Law, F. C.; Rushton, N.; Franks, J. Biocompatibility of Diamond-like Carbon Coating. Biomaterials 1991, 12, 37-40.

(3) Schrand, A. M.; Huang, H.; Carlson, C.; Schlager, J. J.; Ōsawa, E.; Hussain, S. M.; Dai, L. Are Diamond Nanoparticles Cytotoxic? J. Phys. Chem. B 2007, 111, 2-7.

(4) Pope, I.; Payne, L.; Zoriniants, G.; Thomas, E.; Williams, O.; Watson, P.; Langbein, W.; Borri, P. Coherent Anti-Stokes Raman Scattering Microscopy of Single Nanodiamonds. Nat. Nanotechnol. 2014, 9, 940-946.

(5) Hui, Y. Y.; Cheng, C.-L.; Chang, H.-C. Nanodiamonds for Optical Bioimaging. J. Phys. D: Appl. Phys. 2010, 43, 374021.

(6) Mochalin, V. N.; Shenderova, O.; Ho, D.; Gogotsi, Y. The Properties and Applications of Nanodiamonds. Nat. Nanotechnol. 2011, 7, 11-23.

(7) Williams, O. A.; Douhéret, O.; Daenen, M.; Haenen, K.; Ōsawa, E.; Takahashi, M. Enhanced Diamond Nucleation on Monodispersed Nanocrystalline Diamond. Chem. Phys. Lett. 2007, 445, 255-258.

(8) Bautze, T.; Mandal, S.; Williams, O. A.; Rodière, P.; Meunier, T.; Bäuerle, C. Superconducting Nano-Mechanical Diamond Resonators. Carbon 2014, 72, 100-105.

(9) Imboden, M.; Williams, O.; Mohanty, P. Nonlinear Dissipation in Diamond Nanoelectromechanical Resonators. Appl. Phys. Lett. 2013, 102, 103502 .

(10) Mandal, S.; Bautze, T.; Williams, O. A.; Naud, C.; Bustarret, É.; Omnès, F.; Rodière, P.; Meunier, T.; Bäuerle, C.; Saminadayar, L. The Diamond Superconducting Quantum Interference Device. ACS Nano 2011, 5, 7144-7148.

(11) Jelezko, F.; Wrachtrup, J. Single Defect Centres in Diamond: A Review. Phys. Status Solidi A 2006, 203, 3207-3225.

(12) Aharonovich, I.; Castelletto, S.; Simpson, D. A.; Su, C.-H.; Greentree, A. D.; Prawer, S. Diamond-Based Single-Photon Emitters. Rep. Prog. Phys. 2011, 74, 076501.

(13) Becker, J. N.; Görlitz, J.; Arend, C.; Markham, M.; Becher, C. Ultrafast All-Optical Coherent Control of Single Silicon Vacancy Colour Centres in Diamond. Nat. Commun. 2016, 7, 13512.

(14) Balasubramanian, G.; Chan, I. Y.; Kolesov, R.; Al-Hmoud, M.; Tisler, J.; Shin, C.; Kim, C.; Wojcik, A.; Hemmer, P. R.; Krueger, A.; et al. Nanoscale Imaging Magnetometry with Diamond Spins under Ambient Conditions. Nature 2008, 455, 648-651.

(15) Boudou, J. P.; Curmi, P. A.; Jelezko, F.; Wrachtrup, J.; Aubert, P.; Sennour, M.; Balasubramanian, G.; Reuter, R.; Thorel, A.; Gaffet, E. High Yield Fabrication of Fluorescent Nanodiamonds. Nanotechnology 2009, 20, 235602.

(16) Trusheim, M. E.; Li, L.; Laraoui, A.; Chen, E. H.; Bakhru, H.; Schröder, T.; Gaathon, O.; Meriles, C. A.; Englund, D. Scalable Fabrication of High Purity Diamond Nanocrystals with Long-SpinCoherence Nitrogen Vacancy Centers. Nano Lett. 2014, 14, 32-36.

(17) Knowles, H. S.; Kara, D. M.; Atatüre, M. Observing Bulk Diamond Spin Coherence in High-Purity Nanodiamonds. Nat. Mater. 2014, 13, 21-25.

(18) Andrich, P.; Alemán, B. J.; Lee, J. C.; Ohno, K.; de las Casas, C. F.; Heremans, F. J.; Hu, E. L.; Awschalom, D. D. Engineered Microand Nanoscale Diamonds as Mobile Probes for High-Resolution Sensing in Fluid. Nano Lett. 2014, 14, 4959-4964.

(19) Chang, Y.-R.; Lee, H.-Y.; Chen, K.; Chang, C.-C.; Tsai, D.-S.; Fu, C.-C.; Lim, T.-S.; Tzeng, Y.-K.; Fang, C.-Y.; Han, C.-C.; et al. Mass Production and Dynamic Imaging of Fluorescent Nanodiamonds. Nat. Nanotechnol. 2008, 3, 284-288.

(20) Hong, S.; Grinolds, M. S.; Pham, L. M.; Le Sage, D.; Luan, L.; Walsworth, R. L.; Yacoby, A.; Le Sage, D.; Luan, L.; Walsworth, R. L.; et al. Nanoscale Magnetometry with NV Centers in Diamond. MRS Bull. 2013, 38, 155-161.

(21) Yin, Z.-q.; Li, T.; Zhang, X.; Duan, L. M. Large Quantum Superpositions of a Levitated Nanodiamond through Spin-Opto- 
mechanical Coupling. Phys. Rev. A: At., Mol., Opt. Phys. 2013, 88, 033614.

(22) Scala, M.; Kim, M. S.; Morley, G. W.; Barker, P. F.; Bose, S. Matter-Wave Interferometry of a Levitated Thermal Nano-Oscillator Induced and Probed by a Spin. Phys. Rev. Lett. 2013, 111, 180403.

(23) Albrecht, A.; Retzker, A.; Plenio, M. B. Testing Quantum Gravity by Nanodiamond Interferometry with Nitrogen-Vacancy Centers. Phys. Rev. A: At., Mol., Opt. Phys. 2014, 90, 033834.

(24) Bose, S.; Mazumdar, A.; Morley, G. W.; Ulbricht, H.; Toroš, M.; Paternostro, M.; Geraci, A. A.; Barker, P. F.; Kim, M. S.; Milburn, G. Spin Entanglement Witness for Quantum Gravity. Phys. Rev. Lett. 2017, 119, 240401.

(25) Williams, O. A.; Hees, J.; Dieker, C.; Jäger, W.; Kirste, L.; Nebel, C. E. Size-Dependent Reactivity of Diamond Nanoparticles. ACS Nano 2010, 4, 4824-4830.

(26) Rondin, L.; Tetienne, J.-P.; Hingant, T.; Roch, J.-F.; Maletinsky, P.; Jacques, V. Magnetometry with Nitrogen-Vacancy Defects in Diamond. Rep. Prog. Phys. 2014, 77, 056503.

(27) Rahman, A. T. M. A.; Frangeskou, A. C.; Kim, M. S.; Bose, S.; Morley, G. W.; Barker, P. F. Burning and Graphitization of Optically Levitated Nanodiamonds in Vacuum. Sci. Rep. 2016, 6, 21633.

(28) Frangeskou, A. C.; Rahman, A. T. M. A.; Gines, L.; Mandal, S.; Williams, O. A.; Barker, P. F.; Morley, G. W. Pure Nanodiamonds for Levitated Optomechanics in Vacuum. New J. Phys. 2018, 20, 043016.

(29) Volkov, D. S.; Proskurnin, M. A.; Korobov, M. V. Elemental Analysis of Nanodiamonds by Inductively-Coupled Plasma Atomic Emission Spectroscopy. Carbon 2014, 74, 1-13.

(30) Mitev, D. P.; Townsend, A. T.; Paull, B.; Nesterenko, P. N. Direct Sector Field ICP-MS Determination of Metal Impurities in Detonation Nanodiamond. Carbon 2013, 60, 326-334.

(31) Neu, E.; Arend, C.; Gross, E.; Guldner, F.; Hepp, C.; Steinmetz, D.; Zscherpel, E.; Ghodbane, S.; Sternschulte, H.; Steinmüller-Nethl, D.; et al. Narrowband Fluorescent Nanodiamonds Produced from Chemical Vapor Deposition Films. Appl. Phys. Lett. 2011, 98, 243107.

(32) Heyer, S.; Janssen, W.; Turner, S.; Lu, Y.-G.; Yeap, W. S.; Verbeeck, J.; Haenen, K.; Krueger, A. Toward Deep Blue Nano Hope Diamonds: Heavily Boron-Doped Diamond Nanoparticles. ACS Nano 2014, 8, 5757-5764.

(33) Kim, K. H.; Kim, H. Y.; Lim, H. B. Analysis of Si3N4 Ultra Fine Powder Using High-Pressure Acid Digestion and Slurry Injection in Inductively Coupled Plasma Atomic Emission Spectrometry. Bull. Korean Chem. Soc. 2001, 22, 159-163.

(34) Scofield, J. H. Hartree-Slater Subshell Photoionization CrossSections at 1254 and 1487 EV. J. Electron Spectrosc. Relat. Phenom. 1976, 8, 129-137.

(35) Ferro, S.; Dal Colle, M.; De Battisti, A. Chemical Surface Characterization of Electrochemically and Thermally Oxidized BoronDoped Diamond Film Electrodes. Carbon 2005, 43, 1191-1203.

(36) Gardner, S. D.; Singamsetty, C. S. K.; Booth, G. L.; He, G.-R.; Pittman, C. U. Surface Characterization of Carbon Fibers Using Angle-Resolved XPS and ISS. Carbon 1995, 33, 587-595.

(37) Notsu, H. Introduction of Oxygen-Containing Functional Groups onto Diamond Electrode Surfaces by Oxygen Plasma and Anodic Polarization. Electrochem. Solid-State Lett. 1999, 2, 522.

(38) Goeting, C. H.; Marken, F.; Gutiérrez-Sosa, A.; Compton, R. G.; Foord, J. S. Electrochemically Induced Surface Modifications of Boron-Doped Diamond Electrodes: An X-Ray Photoelectron Spectroscopy Study. Diamond Relat. Mater. 2000, 9, 390-396.

(39) Klauser, F.; Ghodbane, S.; Boukherroub, R.; Szunerits, S.; Steinmüller-Nethl, D.; Bertel, E.; Memmel, N. Comparison of Different Oxidation Techniques on Single-Crystal and Nanocrystalline Diamond Surfaces. Diamond Relat. Mater. 2010, 19, 474-478.

(40) Yamamoto, K.; Koga, Y.; Fujiwara, S. XPS Studies of Amorphous SiCN Thin Films Prepared by Nitrogen Ion-Assisted Pulsed-Laser Deposition of SiC Target. Diamond Relat. Mater. 2001, 10, 1921-1926.

(41) Zeppilli, S.; Arnault, J. C.; Gesset, C.; Bergonzo, P.; Polini, R. Thermal Stability and Surface Modifications of Detonation Diamond
Nanoparticles Studied with X-Ray Photoelectron Spectroscopy. Diamond Relat. Mater. 2010, 19, 846-853.

(42) Mérel, P.; Tabbal, M.; Chaker, M.; Moisa, S.; Margot, J. Direct Evaluation of the Sp3 Content in Diamond-like-Carbon Films by XPS. Appl. Surf. Sci. 1998, 136, 105-110.

(43) Jackson, S.; Nuzzo, R. G. Determining hybridization differences for amorphous carbon from the XPS C 1s envelope. Applied Surface Science 1995, 90, 195-203.

(44) Estrade-Szwarckopf, H. XPS Photoemission in Carbonaceous Materials: A "Defect" Peak beside the Graphitic Asymmetric Peak. Carbon 2004, 42, 1713-1721.

(45) Yamashita, T.; Hayes, P. Analysis of XPS Spectra of Fe2+ and Fe3+ Ions in Oxide Materials. Appl. Surf. Sci. 2008, 254, 2441-2449.

(46) Osswald, S.; Yushin, G.; Mochalin, V.; Kucheyev, S. O.; Gogotsi, Y. Control of Sp2/Sp3 Carbon Ratio and Surface Chemistry of Nanodiamond Powders by Selective Oxidation in Air. J. Am. Chem. Soc. 2006, 128, 11635-11642.

(47) Ferrari, A. C.; Robertson, J. Interpretation of Raman Spectra of Disordered and Amorphous Carbon. Phys. Rev. B: Condens. Matter Mater. Phys. 2000, 61, 14095-14107.

(48) Tisler, J.; Balasubramanian, G.; Naydenov, B.; Kolesov, R.; Grotz, B.; Reuter, R.; Boudou, J.-P.; Curmi, P. A.; Sennour, M.; Thorel, A.; et al. Fluorescence and Spin Properties of Defects in Single Digit Nanodiamonds. ACS Nano 2009, 3, 1959-1965.

(49) Davis, M. E.; Chen, Z.; Shin, D. M. Nanoparticle Therapeutics: An Emerging Treatment Modality for Cancer. Nat. Rev. Drug Discovery 2008, 7, 771-782. 